\title{
Subacute cutaneous lupus erythematosus induced by antituberculous drugs
}

\section{Samia Mrabat, Sara Eloudi, Zakia Douhi, Hanane Baybay, Fatima Zahra Mernissi}

Department of Dermatology, University Hospital Hassan II, Fes, Morocco

Corresponding author: Samia Mrabat, MD, E-mail: samiamrabat91@gmail.com

Sir,

Drug-induced lupus erythematosus is an autoimmune disorder usually appearing as a result of chronic exposure to certain drugs (from months to years) and resolving after their cessation. It was first described by Hoffman in 1945, when he reported lupus-like symptoms following sulfadiazine treatment [1]. In 1985, hydrochlorothiazide was reported to induce subacute cutaneous lupus erythematosus (SCLE), thus introducing the concept of drug-induced subacute cutaneous lupus erythematosus [2]. To date, over a hundred drugs from more than ten categories have been implicated in DILE [3]. As with idiopathic lupus, DILE is classified into three forms: systemic DILE, drug-induced subacute cutaneous lupus erythematosus (DISCLE), and chronic cutaneous DILE [4].

A 37-year-old female patient was treated with antituberculosis drugs for the previous four months for tuberculous pleurisy. She noticed asymptomatic erythematous lesions on the face two months after starting the treatment. She had also been complaining of an acute hair loss, arthralgia, and dry-eye and drymouth syndrome. A clinical examination found annular erythematous squamous plaques with clear borders and telangiectatic surfaces sitting at the level of the cheeks and the nose with respect to the nasolabial folds (Fig. 1). Dermoscopy revealed erythema, linear vessels, and keratin plugs (Fig. 2). A skin biopsy confirmed the diagnosis of SCLE by showing vacuolar degeneration of the basal membrane, apoptotic keratinocytes, edema, and a lymphocytic infiltrate in the upper dermis (Fig. 3). Immunological tests were positive for anti-nuclear antibodies, anti-native DNA antibodies, and anti-
SSA/anti-SSB antibodies. Blood work found anemia, lymphopenia, and consumption of complement C3 with no renal failure or proteinuria. The diagnosis of isoniazid-induced lupus was reached, indicating its discontinuation and replacement by a combination of ethambutol and pyrazinamide in addition to rifampicin.

The patient was then treated with hydroxychloroquine and systemic steroids for one year due to the persistence of the arthralgia. However, the rash resolved completely three months after beginning treatment.

Drug-induced SCLE is the most common form of DILE, accounting for $20 \%$ of all SCLE cases [5]. Most patients affected by drug-induced SCLE are female $(72 \%)$, with a mean age of 58 years [4]. SCLE may present weeks to years after the initiation of medication [6]. The lesions begin as erythematous papules/plaques, which progress to

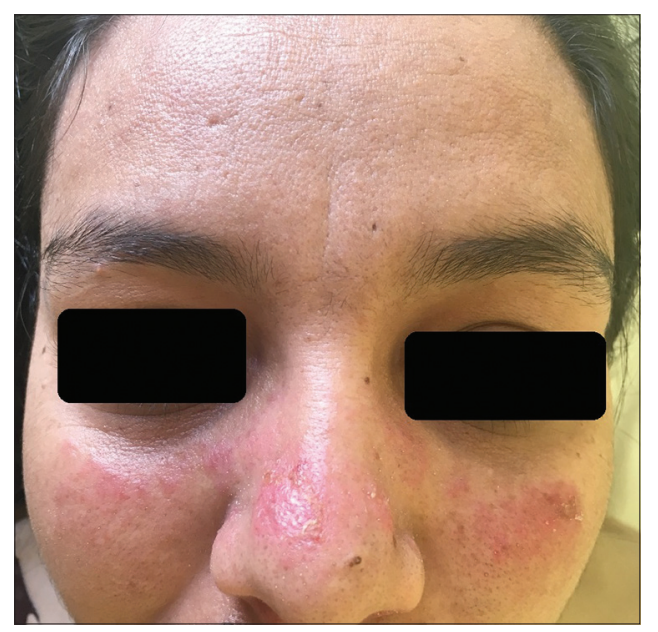

Figure 1: A clinical image showing annular erythematous and squamous plaques with clear borders on the cheeks and the nose with respect to the nasolabial folds.

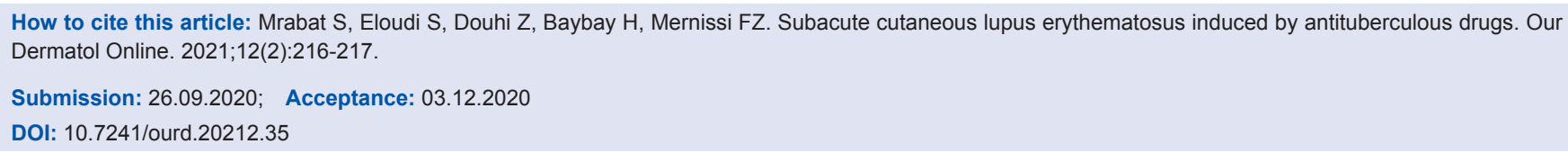




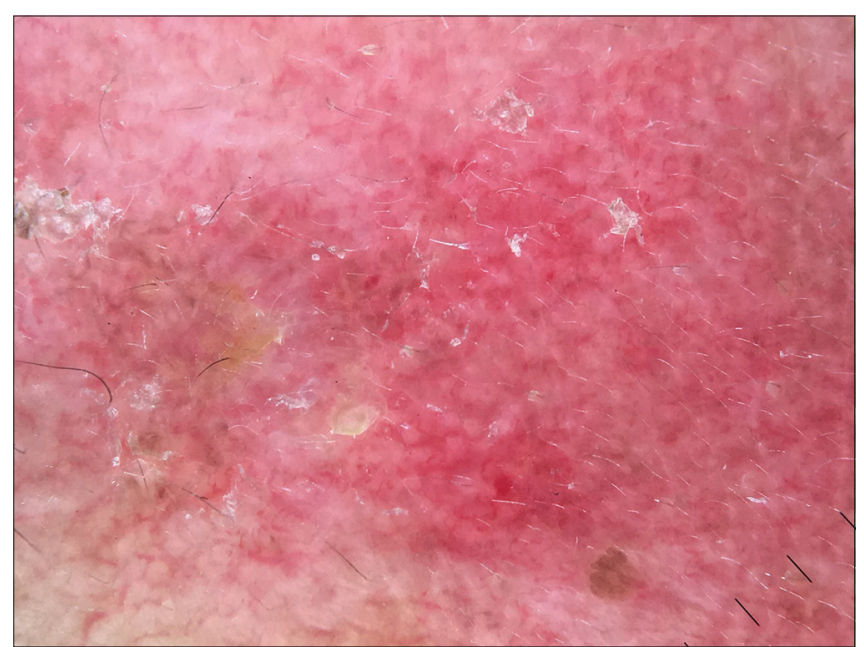

Figure 2: A dermoscopic image revealing the presence of erythema, linear vessels, and keratin plugs.

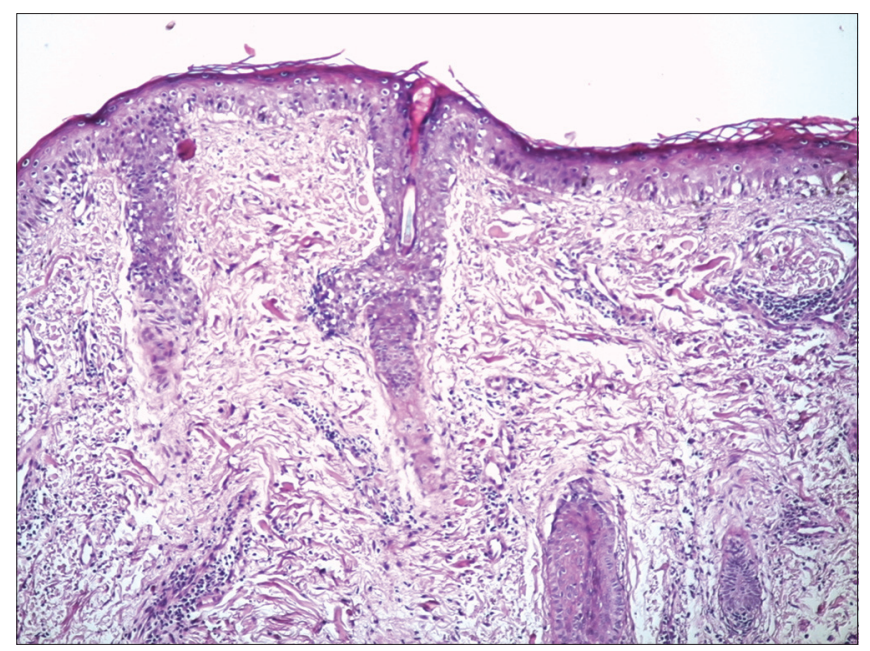

Figure 3: A skin biopsy showing vacuolar degeneration of the basal membrane, apoptotic keratinocytes, and a lymphocytic infiltrate in the upper dermis.

widespread annular, polycyclic lesions with central clearing or papulosquamous lesions. SCLE is strongly associated with the anti-Ro/SSA antibodies (70\% of cases). $60 \%$ to $80 \%$ of cases present positive ANA and $30 \%$ to $50 \%$ present anti-La/SSB antibodies, which was the case in our patient. The patient may also exhibit features of Sjögren syndrome: in our case, the patient complained of dry-eye and dry-mouth syndrome. The criteria for the diagnosis of drug-induced lupus have not been determined. The diagnosis of drug-induced SCLE may be suspected upon characteristic clinical features combined with a relevant drug history supported by positive histopathology and
anti-Ro/SSA antibodies [7]. Regression of the lesions after stopping treatment also allows making the diagnosis of drug-induced lupus.

Spontaneous resolution of DI-SCLE commonly occurs within six to twelve weeks of drug withdrawal. Administration of topical corticosteroids may accelerate the healing process [5]. In our case, isoniazid was interrupted once the diagnosis of DI-SCLE was reached, and the patient was put under topical corticosteroids in addition to hydroxychloroquine and oral steroids for a period of one year. Within three months, the rash resolved completely.

\section{Consent}

The examination of the patient was conducted according to the principles of the Declaration of Helsinki.

The authors certify that they have obtained all appropriate patient consent forms, in which the patients gave their consent for images and other clinical information to be included in the journal. The patients understand that their names and initials will not be published and due effort will be made to conceal their identity, but that anonymity cannot be guaranteed.

\section{REFERENCES}

1. Hoffman BJ. Sensitivity to sulfadiazine resembling acute disseminated lupus erythematosus. Arch Dermatol Syphilol. 1945;51:190-2.

2. Reed BR, Huff JC, Jones SK, Orton PW, Lee LA, Norris DA. Subacute cutaneous lupus erythematosus associated with hydrochlorothiazide therapy. Ann Intern Med. 1985;103:49-51.

3. He Y, Sawalha AH. Drug-induced lupus erythematosus: An update on drugs and mechanisms. Curr Opin Rheumatol. 2018;30:490-7.

4. Dalle Vedove C, Simon JC, Girolomoni G. Drug-induced lupus erythematosus with emphasis on skin manifestations and the role of anti-TNF $\alpha$ agents. J Dtsch Dermatol Ges. 2012;10:889-97.

5. Lowe GC, Henderson CL, Grau RH, Hansen CB, Sontheimer RD. A systematic review of drug-induced subacute cutaneous lupus erythematosus. Br J Dermatol. 2011;164:465-72.

6. Vaglio A, Grayson PC, Fenaroli P, Gianfreda D, Boccaletti V, Ghiggeri GM, Moroni G. Drug-induced lupus: Traditional and new concepts. Autoimmun Rev. 2018;17:912-8.

7. Grönhagen CM, Nyberg F. Cutaneous lupus erythematosus: An update. Indian Dermatol Online J. 2014;5:7-13.

Copyright by Samia Mrabat, et al. This is an open-access article distributed under the terms of the Creative Commons Attribution License, which permits unrestricted use, distribution, and reproduction in any medium, provided the original author and source are credited. Source of Support: Nil, Conflict of Interest: None declared. 